\title{
Hepatopatia esteroidal em gatas após terapia com prednisolona: aspectos laboratoriais, tomográficos e histopatológicos
}

\author{
[Steroid hepatopathy in cats after prednisolone use: laboratory, tomographic, \\ and histopathologic aspects] \\ N.I.C.R. Magalhães ${ }^{1}$, I.C.C. Silva ${ }^{2}$, T.H.T. Fernandes ${ }^{3}$, M.A. Bonelli ${ }^{2}$, \\ M.J.L. Cardoso ${ }^{4}$, V.A. Silva Júnior ${ }^{2}$, F.S. Costa ${ }^{2 *}$ \\ ${ }^{1}$ Faculdade Integrada de Pernambuco (Facipe) - Recife, PE \\ ${ }^{2}$ Universidade Federal Rural de Pernambuco (UFRPE) - Recife, PE \\ ${ }^{3}$ Universidade Maurício de Nassau (Uninassau) - Recife, PE \\ ${ }^{4}$ Universidade Estadual de Londrina (UEL) - Londrina, PR
}

\begin{abstract}
RESUMO
Glicocorticoides são amplamente utilizados na clínica de pequenos animais, entretanto seu uso contínuo pode causar efeitos colaterais. Os gatos são considerados menos susceptíveis a esses efeitos do que outras espécies, mas existem poucos trabalhos abordando os efeitos adversos em felinos. O objetivo deste estudo foi avaliar possíveis alterações laboratoriais, histopatológicas e do grau de atenuação radiográfica do parênquima hepático de gatas submetidas à terapia com prednisolona. Um ensaio clínico foi realizado em quatro gatas hígidas, as quais receberam prednisolona, por via oral, na dose de $3 \mathrm{mg} / \mathrm{kg}$, durante 60 dias consecutivos. Nos achados histopatológicos após 60 dias de tratamento, observou-se desorganização dos cordões de hepatócitos e degeneração vacuolar, além de necrose de hepatócitos, porém não foram observados sinais de fibrose no parênquima hepático. Os dados da tomografia computadorizada demonstram aumento do grau de atenuação do parênquima hepático a partir do $30^{\circ}$ dia da administração de prednisolona, que persistiu até o final do experimento. No presente estudo, foi possível caracterizar a existência de hepatopatia esteroidal em gatos em estágios precoces da terapia com prednisolona.
\end{abstract}

Palavras-chave: felino, glicocorticoide, fígado, hepatopatia, TC

\begin{abstract}
Glucocorticoids are widely used medications in small animal practice; however, its continuous use can have side effects. Cats are considered less susceptible than other species, however, the literature does not usually address adverse effects in felines. The objective of this study was to evaluate possible laboratory and histopathologic changes, as well as changes to the degree of radiographic attenuation of the hepatic parenchyma in cats treated with prednisolone. A clinical trial was done in four healthy cats, who received prednisolone orally at $3 \mathrm{mg} / \mathrm{kg}$ during 60 consecutive days. In the histopathologic findings at 60 days of treatment, there were disorganized hepatocyte chords and vacuolar degeneration, as well as hepatocyte necrosis, however, there were no signs of fibrosis in the hepatic parenchyma. Data obtained via computed tomography showed increase of the degree of attenuation in the hepatic parenchyma from day 30 of prednisolone therapy, which persisted until the end of the experiment. In the present study, it was possible to characterize the existence of steroidal hepathopathy in cats in the early stages of prednisolone therapy.
\end{abstract}

Keyword: feline, glucocorticoid, liver, hepatopathy, CT

Recebido em 14 de junho de 2018

Aceito em 27 de novembro de 2019

*Autor para correspondência (corresponding author)

E-mail: fabianosellos@hotmail.com 


\section{INTRODUÇÃO}

Os glicocorticoides estão entre os fármacos mais frequentemente prescritos na medicina veterinária (Cross et al., 2012) pela ação anti-inflamatória, imunossupressora e antineoplásica (Cross et al., 2012; Lowe, 2016). A prednisolona é um glicocorticoide de ação intermediária (Lowe et al., 2008b), sendo uma droga biologicamente ativa (Bergmann et al., 2012), com biodisponibilidade de $100 \%$ após administração oral e tempo de ação entre 12 e 36 horas (Lowe et al., 2008b). Prednisolona oral na dose entre 2,2 e $8,8 \mathrm{mg} / \mathrm{kg} / \mathrm{dia}$ é recomendada em gatos por sua ação anti-inflamatória e imunossupressora (Lowe et al., 2008a; Lowe, 2016).

Alterações tóxicas e metabólicas resultantes do uso de corticoides em animais são frequentes (Lowe, 2016). Gatos são considerados menos susceptíveis ao desenvolvimento de efeitos colaterais (Cross et al., 2012), porém estudos sobre as ações farmacológicas dos corticoides em gatos são raros (Niessen et al., 2013), e os protocolos terapêuticos são extrapolados de outras espécies e modificados com base na experiência clínica (Lowe, 2016).

A hepatopatia esteroidal em cães causa hepatomegalia, alterações laboratoriais e achados histopatológicos típicos de deposição de glicogênio e vacuolização nos hepatócitos (Costa et al., 2013; Lowe, 2016). Em gatos, é citado que a hepatopatia esteroidal não ocorre (Feldman e Nelson, 2004) ou ocorre em menor frequência (Cross et al., 2012), talvez por ser mais difícil de se detectar (Lowe, 2016).

Lesões hepáticas em gatos apresentam alta morbidade e mortalidade (Lidbury e Suchodolski, 2016). O diagnóstico de hepatopatias é feito com testes laboratoriais, exames de diagnóstico por imagem e exames histopatológicos (Alisson, 2012). A tomografia computadorizada (TC) auxilia no diagnóstico de diversas enfermidades (Kobayashi et al., 2014). A estimativa do grau de atenuação radiográfica pela TC é importante para o diagnóstico e monitoramento de condições que alteram a densidade do órgão (Nakamura et al., 2005; Lam et al., 2014), e tem alta correlação com os achados histopatológicos (Kodama et al., 2007). Dessa forma, o objetivo deste estudo foi avaliar possíveis alterações laboratoriais, tomográficas e histopatológicas do parênquima hepático de gatas submetidas à terapia com prednisolona. A hipótese foi que a corticoterapia seria capaz de promover hepatopatia esteroidal em gatos.

\section{MATERIAL E MÉTODOS}

Os procedimentos relatados neste estudo foram aprovados pelo Comitê de Ética em Uso de Animais (Ceua), sob licença 142/2014. Para a seleção do grupo experimental, foram realizados exame físico, hemograma, urinálise e dosagem dos níveis séricos de alanina aminotransferase (ALT), aspartato aminotransferase (AST), fosfatase alcalina (FA), gamaglutamiltransferase (GGT), ureia, creatinina, glicose, cortisol, hormônio estimulante da tireoide (TSH), T4 livre e T4 total.

O grupo experimental foi composto por quatro gatas adultas jovens (idade variando entre 12 e 36 meses), hígidas, não castradas, sem raça definida e com peso corporal entre 2,5 e $4,0 \mathrm{~kg}$. Primeiramente, as gatas foram submetidas a um período de adaptação de 45 dias, ficando alojadas em baias coletivas com acesso à luz solar, que permitiam atividade física. Todas as gatas receberam água ad libitum e ração premium (Royal Canin ${ }^{\circledR}$ Premium gatos adultos, Descalvado, São Paulo, Brasil) três vezes ao dia.

A fase inicial (momento controle) correspondeu ao primeiro dia do experimento, sendo realizados TC, dosagem dos níveis séricos sanguíneos e análise histopatológica antes de se iniciar a prednisolona. A prednisolona foi, então, administrada por via oral, a cada 24 horas, a uma dose de $3 \mathrm{mg} / \mathrm{kg}$. No $30^{\circ}$ dia da administração, foram realizados TC e dosagem dos níveis séricos da ALT, AST, GGT, FA, ureia, creatinina e glicose. A prednisolona foi administrada durante 60 dias. No último dia (fase final do protocolo experimental), foram realizados os mesmos exames do momento controle. As gatas foram pesadas semanalmente, e as doses administradas de prednisolona ajustadas quando necessário. Após o final do período experimental, a dose de prednisolona foi gradualmente reduzida em todas as gatas.

Para os exames de TC, as gatas foram submetidas a jejum hídrico de quatro horas e alimentar de oito horas. Para a realização de TC, foi feita fluidoterapia intravenosa (IV) com solução fisiológica em velocidade de infusão de $10 \mathrm{~mL} / \mathrm{kg} / \mathrm{h}$, para administração de medicação pré- 
anestésica diazepam $(0,5 \mathrm{mg} / \mathrm{kg})$ e indução anestésica com propofol $(0,4 \mathrm{mg} / \mathrm{kg})$. A manutenção anestésica foi feita com isoflurano diluído em oxigênio $100 \%$. As gatas foram posicionadas em decúbito ventral.

Os exames de TC foram feitos com tomógrafo helicoidal (GE Hi-Speed FXI, Waukesha, Wisconsin, Estados Unidos) e protocolo com 120 $\mathrm{kVp}$ e mA automático na velocidade de uma rotação por segundo, pitch de 1 e diâmetro de matriz de 512 x 512 pixels. Primeiramente foram obtidas imagens sem contraste e, em seguida, com administração de contraste iodado intravenoso (ioversol, $2 \mathrm{~mL} / \mathrm{kg}$ ). Foram obtidos cortes transversais de $2 \mathrm{~mm}$ de espessura, no sentido craniocaudal, desde a cúpula diafragmática até as cristas ilíacas, com intervalos entre cortes de $1 \mathrm{~mm}$ e com filtro para partes moles. Antes dos exames, o aparelho foi devidamente calibrado para melhor padronização dos resultados.

Após digitalização das imagens, calculou-se o valor de atenuação em unidades Hounsfield (HU) do fígado com base na média de três regiões de interesse do parênquima (ROI - regions of interest) e do baço a partir de um ROI. Estruturas vasculares não foram incluídas nos ROIs. Cada ROI foi selecionado manualmente por um único observador (F.S.C.), tendo uma área circular de 25 $\pm 0,1 \mathrm{~mm}^{2}$. A avaliação da radiodensidade hepática e esplênica foi realizada em três diferentes níveis de corte dos órgãos, totalizando nove ROIs para a análise hepática e três ROIs para a análise esplênica em cada gata. Foram selecionados ROIs no lobo lateral esquerdo, no lobo lateral direito e no lobo quadrado do fígado (Fig. 1), seguindo-se metodologia para humanos (Kodama et al., 2007), e a segmentação do órgão baseou-se na posição da veia cava caudal e das veias hepáticas de cada gata. As imagens contrastadas ajudaram a delimitar a vascularização do fígado e a definir o ponto de análise. Para o baço, a medida foi feita na região de maior volume em cada nível de corte. Para determinação do grau de atenuação do fígado e do baço e razão entre esses órgãos, utilizaram-se as imagens sem contraste. As imagens foram avaliadas utilizando-se software comercial de visualização de imagens DICOM (OsiriX-64 bits, versão 7.0).

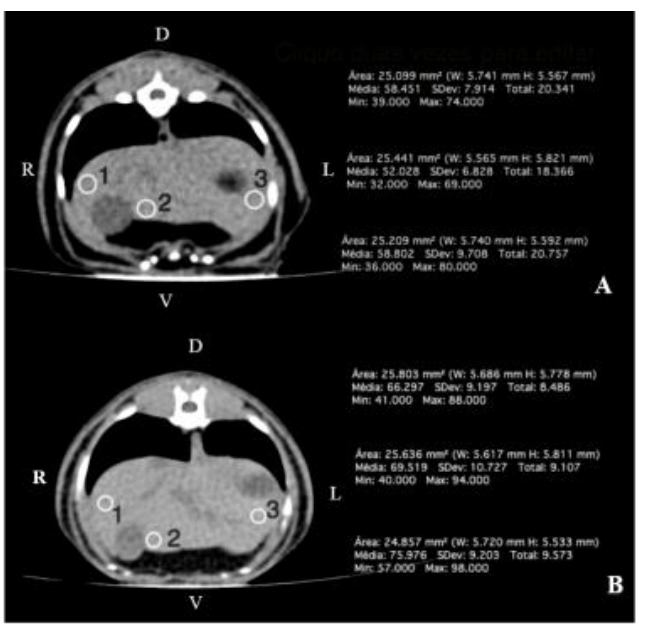

Figura 1. Pontos de medidas de atenuação radiográfica hepática em gata hígida antes (imagem A) e depois da administração oral de prednisolona (imagem B). 1 - lobo lateral direito, 2 - lobo quadrado, 3 - lobo lateral esquerdo. D: dorsal; V: ventral; L: esquerdo; R: direito.

Após a TC, realizou-se biopsia incisional do fígado das gatas, sendo coletados fragmentos da porção periférica dos lobos direito e esquerdo de cada gata antes e depois da administração da prednisolona, respectivamente. As amostras foram fixadas em formol tamponado, processadas de acordo com as técnicas de rotina para parafina e coradas pela hematoxilina-eosina (HE), coloração de ácido periódico de Schiff (PAS) e tricrômico de Mallory.

O grau de severidade da degeneração vacuolar dos hepatócitos das gatas tratadas com prednisolona durante 60 dias foi classificado de forma similar ao descrito por Lowe et al. (2008a), em que no grau 0 , os hepatócitos não apresentam degeneração vacuolar; no grau 1, os hepatócitos apresentam degeneração vacuolar em até 33\% do lobo; no grau 2, apresentam envolvimento entre 34 e $66 \%$ do lobo. e no grau 3, a degeneração vacuolar atinge mais de $66 \%$ do lobo.

Análise descritiva dos dados foi feita mediante distribuições absolutas e relativas para as variáveis categóricas. Média, desvio-padrão e coeficiente de variação para as variáveis numéricas foram analisados inferencialmente por meio dos testes de Friedman e Wilcoxon para dados pareados. A margem de erro utilizada foi de $5 \%$, e os intervalos foram obtidos com $95 \%$ de confiabilidade. 


\section{RESULTADOS}

Verificou-se diferença estatística $(\mathrm{P}<0,05)$ entre os tempos de avaliação para as variáveis: peso, ALT, AST e glicose (Tab. 1). A média do peso aumentou em 30 dias e 60 dias em comparação com o momento inicial. Os valores médios da ALT aumentaram com o tempo de avaliação, tendo o maior aumento ocorrido de 30 dias para 60 dias. A média da AST na avaliação inicial foi de $27,18 \mathrm{UI} / \mathrm{L}$ e variou de 84,50 a $85,15 \mathrm{UI} / \mathrm{L}$ com 30 e 60 dia,s respectivamente, sendo verificada diferença estatística entre a avaliação inicial e com 30 dias de prednisolona e entre a avaliação inicial e com 60 dias de prednisolona. A glicose aumentou após 30 dias de prednisolona $(200,88 \mathrm{~g} / \mathrm{dL})$ comparada com o valor inicial $(91,75 \mathrm{~g} / \mathrm{dL})$, mas diminuiu aos 60 dias $(137,00 \mathrm{~g} / \mathrm{dL})$ comparada com o $30^{\circ}$ dia.

Tabela 1. Medidas de tendência central do peso e marcadores bioquímicos séricos de quatro gatas antes e após administração de prednisolona por 60 dias

\begin{tabular}{|c|c|c|c|c|}
\hline Variável & Momento & Média & $\mathrm{DP}$ & $\mathrm{CV}$ \\
\hline Peso (kg) & $\begin{array}{l}\text { Inicial } \\
30 \text { dias } \\
60 \text { dias }\end{array}$ & $\begin{array}{l}3,13 \\
3,44 \\
3,95\end{array}$ & $\begin{array}{l}0,75 \\
0,81 \\
0,72\end{array}$ & $\begin{array}{l}24,00 \\
23,44 \\
18,20\end{array}$ \\
\hline $\operatorname{ALT}(\mathrm{UI} / \mathrm{L})$ & $\begin{array}{l}\text { Inicial } \\
30 \text { dias } \\
60 \text { dias }\end{array}$ & $\begin{array}{l}57,03 \\
62,00 \\
118,28\end{array}$ & $\begin{array}{l}7,31 \\
14,39 \\
19,46\end{array}$ & $\begin{array}{l}12,83 \\
23,20 \\
16,45\end{array}$ \\
\hline AST (UI/L) & $\begin{array}{l}\text { Inicial } \\
30 \text { dias } \\
60 \text { dias }\end{array}$ & $\begin{array}{l}27,18 \\
84,50 \\
85,15\end{array}$ & $\begin{array}{l}4,54 \\
20,96 \\
12,06\end{array}$ & $\begin{array}{l}16,71 \\
24,81 \\
14,17\end{array}$ \\
\hline FA (UI/L) & $\begin{array}{l}\text { Inicial } \\
30 \text { dias } \\
60 \text { dias }\end{array}$ & $\begin{array}{l}54,75 \\
45,18 \\
46,75\end{array}$ & $\begin{array}{l}3,86 \\
9,09 \\
22,26\end{array}$ & $\begin{array}{l}7,05 \\
20,12 \\
47,62\end{array}$ \\
\hline GGT(U/I) & $\begin{array}{l}\text { Inicial } \\
30 \text { dias } \\
60 \text { dias }\end{array}$ & $\begin{array}{l}2,58 \\
1,18 \\
3,08\end{array}$ & $\begin{array}{l}0,51 \\
0,10 \\
1,95\end{array}$ & $\begin{array}{l}19,90 \\
8,15 \\
63,47\end{array}$ \\
\hline Ureia (mg/dL) & $\begin{array}{l}\text { Inicial } \\
30 \text { dias } \\
60 \text { dias }\end{array}$ & $\begin{array}{l}54,58 \\
46,38 \\
47,30\end{array}$ & $\begin{array}{l}5,91 \\
1,56 \\
2,56\end{array}$ & $\begin{array}{l}10,82 \\
3,37 \\
5,40\end{array}$ \\
\hline Creatinina $(\mathrm{g} / \mathrm{dL})$ & $\begin{array}{l}\text { Inicial } \\
30 \text { dias } \\
60 \text { dias }\end{array}$ & $\begin{array}{l}1,28 \\
1,08 \\
1,08\end{array}$ & $\begin{array}{l}0,10 \\
0,10 \\
0,25\end{array}$ & $\begin{array}{l}7,51 \\
8,91 \\
23,26\end{array}$ \\
\hline Glicose (g/dL) & $\begin{array}{l}\text { Inicial } \\
30 \text { dias } \\
60 \text { dias }\end{array}$ & $\begin{array}{l}91,75^{(\mathbf{A})} \\
200,88^{(\mathbf{B})} \\
137,00^{\text {(C) }}\end{array}$ & $\begin{array}{l}4,65 \\
35,21 \\
30,60\end{array}$ & $\begin{array}{l}5,06 \\
17,53 \\
22,34\end{array}$ \\
\hline Cortisol (mcg/dL) & $\begin{array}{l}\text { Inicial } \\
60 \text { dias }\end{array}$ & $\begin{array}{l}2,25 \\
0,51\end{array}$ & $\begin{array}{l}0,31 \\
0,22\end{array}$ & $\begin{array}{l}13,82 \\
43,10\end{array}$ \\
\hline TSH (ng/mL) & 60 dias & $\begin{array}{l}0,17 \\
0,17\end{array}$ & $\begin{array}{l}0,04 \\
0,08\end{array}$ & $\begin{array}{l}21,48 \\
47,66\end{array}$ \\
\hline T4 livre (ng/dL) & $\begin{array}{l}\text { Inicial } \\
60 \text { dias }\end{array}$ & $\begin{array}{l}2,68 \\
1,83\end{array}$ & $\begin{array}{l}0,59 \\
0,27\end{array}$ & $\begin{array}{l}21,97 \\
14,81\end{array}$ \\
\hline T4 total (ng/dL) & $\begin{array}{l}\text { Inicial } \\
60 \text { dias }\end{array}$ & $\begin{array}{l}29,28 \\
25,53\end{array}$ & $\begin{array}{l}2,49 \\
10,00\end{array}$ & $\begin{array}{l}8,50 \\
39,18\end{array}$ \\
\hline
\end{tabular}

ALT - alanina aminotransferase; AST - aspartato aminotransferase; FA - fosfatase alcalina; GGT gamaglutamiltransferase; TSH - hormônio estimulante da tireoide; T4 livre - tiroxina livre; T4 total - tiroxina total; DP - desvio-padrão; CV - coeficiente de variação. 
Verificou-se diferença estatística entre os tempos de avaliação dos valores médios de radiodensidade do fígado e entre os lobos hepáticos (Tab. 2). Ressalta-se que, no fígado e em cada lobo hepático avaliado, a média de atenuação radiográfica foi menor na avaliação inicial, apresentando-se mais elevada na avaliação com 30 dias, seguida da avaliação com 60 dias. Os testes de comparações múltiplas mostraram diferenças estatísticas significativas, exceto entre os tempos de avaliação com 30 dias e 60 dias do fígado e dos lobos hepáticos quadrado e esquerdo. Não houve diferença entre os tempos de avaliação do baço e da relação fígado/baço em cada tempo de avaliação $(\mathrm{P}>0,05)$.

Tabela 2. Medidas de atenuação radiográfica em unidades Hounsfield (HU) e a relação entre o tempo de avaliação do fígado e do baço antes e durante administração de prednisolona por 60 dias, em quatro gatas

\begin{tabular}{|c|c|c|c|c|}
\hline Variável & $\begin{array}{l}\text { Tempo de } \\
\text { avaliação }\end{array}$ & Média & DP & $\mathrm{CV}$ \\
\hline \multirow[t]{3}{*}{ Lobo hepático direito } & Inicial & 54,17 & 1,90 & 3,50 \\
\hline & 30 dias & 70,25 & 4,41 & 6,28 \\
\hline & 60 dias & 63,92 & 3,10 & 4,84 \\
\hline \multirow[t]{3}{*}{ Lobo hepático quadrado } & Inicial & 53,17 & 2,94 & 5,53 \\
\hline & 30 dias & 67,92 & 6,43 & 9,47 \\
\hline & 60 dias & 64,08 & 3,46 & 5,40 \\
\hline \multirow{3}{*}{ Lobo hepático esquerdo } & Inicial & 53,33 & 4,01 & 7,52 \\
\hline & 30 dias & 67,67 & 5,64 & 8,33 \\
\hline & 60 dias & 63,33 & 3,66 & 5,78 \\
\hline \multirow[t]{3}{*}{ Fígado } & Inicial & 53,56 & 2,73 & 5,10 \\
\hline & 30 dias & 68,61 & 5,32 & 7,75 \\
\hline & 60 dias & 63,78 & 2,97 & 4,66 \\
\hline \multirow[t]{3}{*}{ Baço } & Inicial & 51,83 & 2,15 & 4,15 \\
\hline & 30 dias & 51,58 & 5,29 & 10,26 \\
\hline & 60 dias & 50,67 & 5,01 & 9,89 \\
\hline \multirow[t]{3}{*}{ Relação do fígado/baço } & Inicial & 1,03 & 0,07 & 6,80 \\
\hline & 30 dias & 1,34 & 0,15 & 11,19 \\
\hline & 60 dias & 1,27 & 0,15 & 11,81 \\
\hline
\end{tabular}

A análise histopatológica do fígado na fase inicial revelou os hepatócitos preservados e estando de modo radiado em relação à veia central (Fig. 2A). Entretanto, no fígado das gatas com 60 dias de prednisolona, foi constatada congestão, desorganização dos cordões de hepatócitos, degeneração vacuolar, necrose de hepatócitos e infiltrado mononuclear linfocitário (Fig. 2B), além de deposição de colágeno ao redor da veia centro lobular e espaço porta (Fig. 2C). A degeneração vacuolar dos hepatócitos com 60 dias de corticoterapia foi classificada em grau 3, de acordo com a metodologia descrita por Lowe et al. (2008a).

\section{DISCUSSÃO}

Verificou-se a existência de hepatopatia esteroidal no grupo experimental. Poucos estudos relatam a ocorrência de hepatopatia secundária à administração de corticoides em gatos (Ferasin, 2001; Lowe et al., 2008a), mas alguns autores questionam sua existência (Feldman e Nelson, 2004). Hepatopatia relacionada ao hiperadrenocorticismo iatrogênico em gatos foi sugerida em dois casos devido ao aumento da atividade das enzimas hepáticas e hepatomegalia (Ferasin, 2001). 

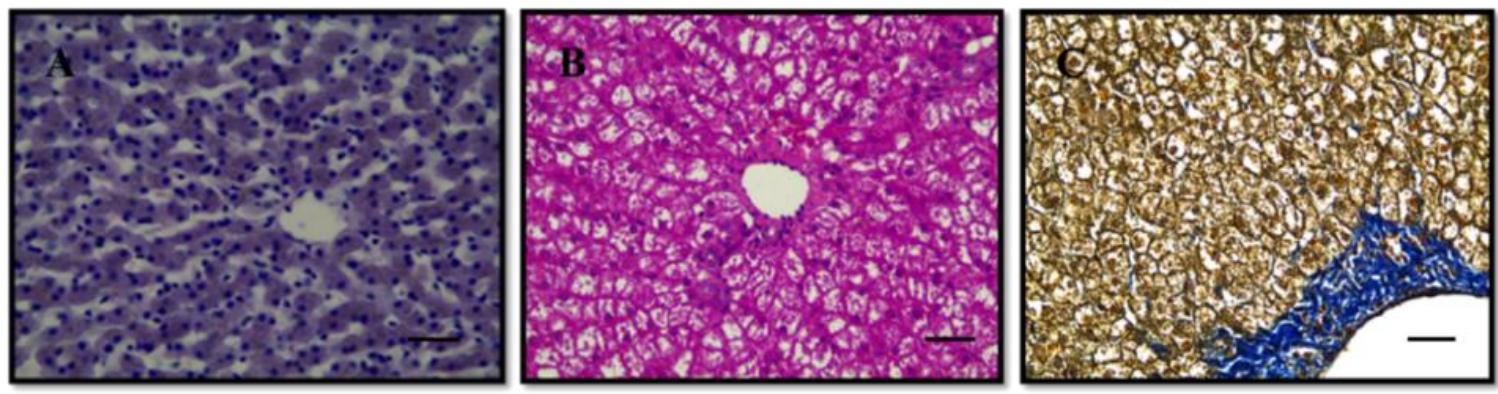

Fig. 2. Fotomicrografia do fígado de gatas antes e durante administração de prednisolona por 60 dias. (A) fase inicial, $(\mathrm{HE})$ : observar os hepatócitos em forma de fileiras ou cordões de forma radiada à veia central. (B) fase final - 60 dias com prednisolona, (HE), sinais de desorganização dos cordões de hepatócitos e sinais de degeneração vacuolar e necrose de hepatócitos. (C) fase final - 60 dias com prednisolona,

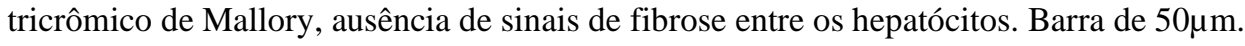

Em cães, a administração de glicocorticoides, mesmo por curtos períodos, resulta em alterações hepáticas (Abdou et al., 2013; Costa et al., 2013; Sobczak-Filipiak et al., 2014), caracterizadas por vacuolização citoplasmática dos hepatócitos e aumento da atividade sérica de FA, GGT e ALT (Abdou et al., 2013; Chapman e Hostutler, 2013; Costa et al., 2013). Alterações ultrassonográficas (Abdou et al., 2013) e tomográficas (Costa et al., 2013) também já foram descritas em cães.

O hiperadrenocorticismo espontâneo em gatos é raro (Gunn-Moore, 2005; Cross et al., 2012; Niessen et al., 2013; Valentin et al., 2014), e existem poucas informações sobre os achados clínicos, laboratoriais e de diagnóstico por imagem (Cross et al., 2012; Valentin et al., 2014). Um estudo retrospectivo de 30 gatos com hiperadrenocorticismo espontâneo identificou alterações ultrassonográficas, incluindo hepatomegalia e hiperecogenicidade do parênquima, no fígado de 14 animais (Valentin et al., 2014).

Perda de peso é um achado frequente em gatos com hiperadrenocorticismo (Feldman e Nelson, 2004) devido aos efeitos catabólicos do excesso de cortisol (Lowe et al., 2008b). Entretanto, um aumento do peso foi verificado no presente experimento, fato também descrito no hiperadrenocorticismo iatrogênico (Ferasin, 2001). Valentin e colaboradores (2014) observaram que $23 \%$ dos gatos com hiperadrenocorticismo apresentaram ganho de peso. O curto período de administração de prednisolona no presente estudo pode estar relacionado ao ganho de peso por indução à polifagia.
Quanto à hiperglicemia observada, a maioria dos gatos com hiperadenocorticismo apresentam diabetes mellitus (Niessen et al., 2013) devido à maior sensibilidade aos efeitos diabetogênicos pelo excesso de cortisol (Lowe et al., 2008a), diferentemente da espécie canina, que só desenvolve diabetes mellitus em casos mais graves de hiperadrenocorticismo. Corticosteroides podem diminuir a sensibilidade da insulina em gatos (Lowe et al., 2009; Rand, 2013; Lowe, 2016), porém não existem descrições detalhadas da taxa de reposição de glicose e fluxos metabólicos em gatos diabéticos. Supõe-se que a sensibilidade à insulina do fígado seja acompanhada de um aumento da produção endógena de glicose a partir do estímulo à glicogenólise, como em humanos (Lowe et al., 2008b). Glicorticoides antagonizam os efeitos da insulina no fígado e aumentam a produção de glicose (Lowe et al., 2008b; Lowe, 2016). A síntese de glicogênio também é estimulada pela inibição da enzima glicogênio-fosforilase e ativação da glicogênio-sintase (Schake et al., 2002).

As alterações na atividade sérica das enzimas ALT e AST também ocorrem no hiperadrenocorticismo felino (Gunn-Moore, 2005). A atividade sérica da FA não foi alterada nos gatos deste estudo; em cães, entretanto, ocorre aumento de FA no hiperadrenocorticismo (Lowe et al., 2008b; Cross et al., 2012; Niessen et al., 2013; Lowe, 2016). Essa diferença pode ser justificada pela carência da isoenzima da fosfatase alcalina induzida por corticoides em gatos (Lowe et al., 2008b; Cross et al., 2012; Lowe, 2016). Por esse motivo, qualquer elevação da atividade sérica 
da FA total em gatos deve ser considerada clinicamente relevante (Cross et al., 2012).

A análise tomográfica tem sido amplamente utilizada na medicina humana para o diagnóstico de hepatopatias difusas, tais como esteatose ou acúmulo de glicogênio hepatocelular (Festi et al., 2013), o que minimiza a realização de biopsias (Davidson et al., 2006; Kodama et al., 2007). Na medicina veterinária, a utilização da TC para diagnóstico de hepatopatias difusas é descrita em cães (Costa et al., 2013), gatos (Nakamura et al., 2005; Lam et al., 2014) e quelônios (Marchiori et al., 2015).

O aumento da atenuação radiográfica do parênquima hepático, verificado por meio das análises dos ROIs e em razão da atenuação entre fígado e baço, ocorreu em todas as gatas de forma difusa e similar em todos os lobos hepáticos. As análises histopatológicas demonstraram alterações hepatocelulares compatíveis com degeneração vacuolar semelhantes ao observado em gatos tratados com glicocorticoides (Lowe et al., 2008a). Em humanos, o acúmulo de glicogênio hepático é a principal causa de fígados hiperatenuantes, e estudos in vitro demonstram que, para cada aumento de $1 \%$ da concentração de glicogênio hepático, ocorre um aumento do coeficiente de atenuação de raios-X entre 2,5 e $3,0 \mathrm{HU}$ na TC (Tsujimoto et al., 2006). Especificamente em gatos, uma diminuição proporcional da gordura em relação ao glicogênio hepacelular parece ser a causa do aumento do grau de atenuação radiográfica do fígado.

Como limitações desta pesquisa, destaca-se que múltiplas variáveis podem afetar a atenuação radiográfica, tais como $\mathrm{Kvp}, \mathrm{mA}$, algoritmo de reconstrução, espessura de corte, entre outras (Lam et al., 2014). Entretanto, acredita-se que a interferência desses fatores tenha sido baixa, pois os exames foram realizados nas mesmas gatas, em momentos diferentes, utilizando-se o mesmo aparelho e protocolo tomográfico em todos os estudos, com calibração prévia do tomógrafo.

Pode-se concluir que o uso de prednisolona a cada 24 horas, por via oral, na dose de $3,0 \mathrm{mg} / \mathrm{kg}$, promove hepatopatia esteroidal em gatos, desencadeando aumento na atividade sérica de ALT e AST, hiperglicemia, aumento da atenuação radiográfica do parênquima hepático no exame tomográfico e alterações histopatológicas importantes. Apesar de os gatos serem considerados menos susceptíveis aos efeitos colaterais dos glicocorticoides, sugere-se cautela e monitoramento dos pacientes quando necessária a administração de altas doses, mesmo por curtos períodos de tratamento.

\section{AGRADECIMENTOS}

À Coordenação de Aperfeiçoamento de Pessoal de Nível Superior (Capes) pelo auxílio financeiro.

\section{REFERÊNCIAS}

ALLISON, R.W. Laboratory evaluation of the liver. In: Thrall, M.A.G.; WEISER, R.; ALLISON, W.; CAMPBELL, T.W. (Eds) Veterinary hematology and clinical chemistry. Hoboken: Wiley Blackwell, 2012, p. 401-424.

ABDOU, O.A.; KELANY, W.M.; TORAD, F.A.; YEHIA, S.G. Ultrasonographic, morphologic and biochemical alterations in experimentally induced steroid hepatopathy in dogs. Global Vet., v.11, p.123-130, 2013.

BERGMANN, T.K.; BARRACLOUGH, K.A.; LEE, K.J.; STAATZ, C.E. Clinical pharmacokinetics and pharmacodynamics of prednisolone and prednisone in solid organ transplantation. Clin. Pharmacokinet., v.51, p.711-741, 2012.

CHAPMAN, S.E.; HOSTUTLER, R.A. A laboratory diagnostic approach to hepatobiliary disease in small animals. Vet. Clin. N. Am. Food Anim. Pract., v. 43, p.1209-1225, 2013.

COSTA, L.A.V.S.; OLIVEIRA, D.C.; LOPES, B.F. et al. Quantitative computed tomography of the liver in dogs submitted to prednisone therapy. Arq. Bras. Med. Vet. Zootec., v.65, p.1084-1090, 2013.

CROSS, E.; MORELAND, R.; WALLACK, S. Feline pituitary-dependent hyperadrenocorticism and insulin resistance due to a plurihormonal adenoma. Top Companion Anim. Med., v.27, p.820, 2012.

DAVIDSON, L.E.; KUK, J.L.; CHURCH, T.S.; ROSS, R. Protocol for measurement of liver fat by computed tomography. J. Appl. Phycol., v.100, p.864-868, 2006. 
FELDMAN, E.C.; NELSON, R.W. Glucocorticoid therapy. In: FELDMAN, E.C.; NELSON, R.W. (Eds.). Canine and feline endocrinology and reproduction. 3.ed. St Louis, W.B.: Saunders, 2004. p.464-483.

FERASIN, L. Iatrogenic hyperadrenocorticism in a cat following a short therapeutic course of methylprednisolone acetate. J. Feline Med. Surg., v.3, p.87-93, 2001.

FESTI, D.; SCHIUMERINI, R.; MARZI, L.; DI BIASE, A.R. et al. Review article: the diagnosis of non-alcoholic fatty liver disease - availability and accuracy of non-invasive methods. Aliment. Pharmacol. Ther., v.37, p.392-400, 2013.

GUNN-MOORE, D. Feline endocrinopathies. Vet. Clin. Small Anim., v.35, p.171-210, 2005.

KOBAYASHI, T.; KOIE, H.; KUSUMI, A.; KITAGAWA, M. et al. Comparative investigation of body composition in male dogs using CT and body fat analysis software. J. Vet. Med. Sci., v.76, p.439-446, 2014.

KODAMA, Y.; NG, C.S.; WU, T.T.; AYERS, G.D. et al. Comparison of CT methods for determining the fat content of the liver. AJR Am. J. Roentgenol., v.188, p.1307-1321, 2007.

LAM, R.; NIESSEN, S.J.; LAMB, C. X- ray attenuation of the liver and kidney in cats considered at varyng risk of hepatic lipidosis. Vet. Radiol. Ultrasound, v.55, p.141-146, 2014.

LIDBURY, J.A.; SUCHODOLSKI, J.S. New advances in the diagnosis of canine and feline liver and pancreatic disease. Vet. J., v.215, p.8795, 2016.

LOWE, A.D. Glucocorticoids in feline dermatology. In: LITTHE, S. August's consultation in feline internal medicine. [s.l.]: Elsevier, 2016. v.7, p.326-333.

LOWE, A.D.; CAMPBELL, K.L.; BARGER, A.; SCHAEFFER, D.J.; BORST, L. Clinical, clinicopathological and histological effects observed in 14 cats treated with glucocorticoids. Vet. Rec., v.162, p.777-783, 2008a.

LOWE, A.D.; CAMPBELL, K.L.; GRAVES, T. Glucocorticoids in the cat. Vet. Dermatol., v.19, p.340-347, 2008b.
LOWE, A.D.; GRAVES, T.K.; CAMPBELL, K.L.; SCHAEFFER, D.J. a pilot study comparing the diabetogenic effects of dexamethasone and prednisolone in cats. J. Am. Anim. Hosp. Assoc., v.45, p.215-224, 2009.

MARCHIORI, A.; SILVA, I.C.C.; BONELLI, M.A.; ZANOTTI, L.C.R.A. et al. Use of computed tomography for investigation of hepatic lipidosis in captive Chelonoidis carbonaria (SPIX, 1824). J. Zoo Wildl. Med., v.46, p.320-324, 2015.

NAKAMURA, M.; CHEN, H.M.; MOMOI, Y.; IWASAKI, T. Clinical application of computed tomography for the diagnosis of feline hepatic lipidosis. J. Vet. Med. Sci., v.67, p.1163-1165, 2005.

NIESSEN, S.J.M.; CHURCH, D.B.; FORCADA, Y. Hypersomatotropism, acromegaly, and hyperadrenocorticism and feline. Vet. Clin. N. Am. Small Anim. Pract., v.43, p.319-350, 2013.

RAND, J. Pathogenesis of feline diabetes. Vet. Clin. N. Am. Food Anim. Pract., v.43, p.221-231, 2013.

SCHACKE, H.; DOCKE, W.; ASADULLAH, K. Mechanisms involved in side effects of glucocorticoids. Pharmacol. Ther., v.96, p.23-43, 2002.

SOBCZAK-FILIPIAK, M.; SZAREK, J.; CZOPOWICZ, M.; MIECZKOWSKA, J.; LECHOWSKI, R. Hepatic stellate cells in the liver of dogs with steroid-induced hepatopathy. Bull. Vet. Inst. Pulawy, v.58, p.273-276, 2014.

TSUJIMOTO, T.; TAKANO, M.; NISHIOFUKU, M.; YOSHIJI, H. et al. Rapid onset of glycogen storage hepatomegaly in a type2 diabetic patient after a massive dose of longacting insulin and large doses of glucose. Internal. Med., v.45, p.469-473, 2006.

VALENTIN, S.Y.; CORTRIGHT, C.C.; NELSON, R.W.; PRESSLER, B.M. et al. Clinical findings, diagnostic test results, and treatment outcome in cats with spontaneous hyperadrenocorticism: 30 cases. J. Vet. Intern. Med., v.28, p.481-487, 2014. 